Supporting Information

\title{
Weak Van der Waal Stacking, Wide-Range Band Gap and Raman Study on Ultrathin Layers of Metal Phosphorus Trichalcogenides
}

\author{
Ke-zhao Du, ${ }^{\text {a, } b, \ddagger}$ Xing-zhi Wang, ${ }^{b, \ddagger}$ Yang Liu,,${ }^{\text {b, }}$ Peng Hu, ${ }^{a}$ M. Iqbal Bakti Utama, ${ }^{b}$ \\ Chee Kwan Gan, ${ }^{d}$ Qihua Xiong, ${ }^{b, c, *}$ Christian Kloc ${ }^{\text {a, * }}$
}

${ }^{a}$ School of Materials Science \& Engineering, Nanyang Technological University, 639798, Singapore.

${ }^{\mathrm{b}}$ Division of Physics and Applied Physics, School of Physical and Mathematical Sciences, Nanyang Technological University, 637371, Singapore.

c NOVITAS, Nanoelectronics Centre of Excellence, School of Electrical and Electronic Engineering, Nanyang Technological University, 639798, Singapore.

${ }^{d}$ Institute of High Performance Computing, Agency for Science, Technology and Research, 1 Fusionopolis Way, \#16-16 Connexis, Singapore 138632, Singapore..

Chemical: Cadmium powder (-200 mesh, 99.99\%), zinc flake (-325 mesh, 99.9\%), manganese granule (0.03-0.47 in, 99.98\%), red phosphorus lump (puratronic, 99.999+\%), sulfur pieces (Puratronic, 99.9995\%), selenium powder (200 -mesh, 99.999\%) and iodine spheres (ultra dry, 10 -mesh, 99.998\%) were all purchased from the Alfa Aesar. Nickel (nanosize activated powder, $99.9+\%$ ) and iron (fine powder, $\geqslant 99 \%$ ) were purchased from the Sigma-Aldrich. 

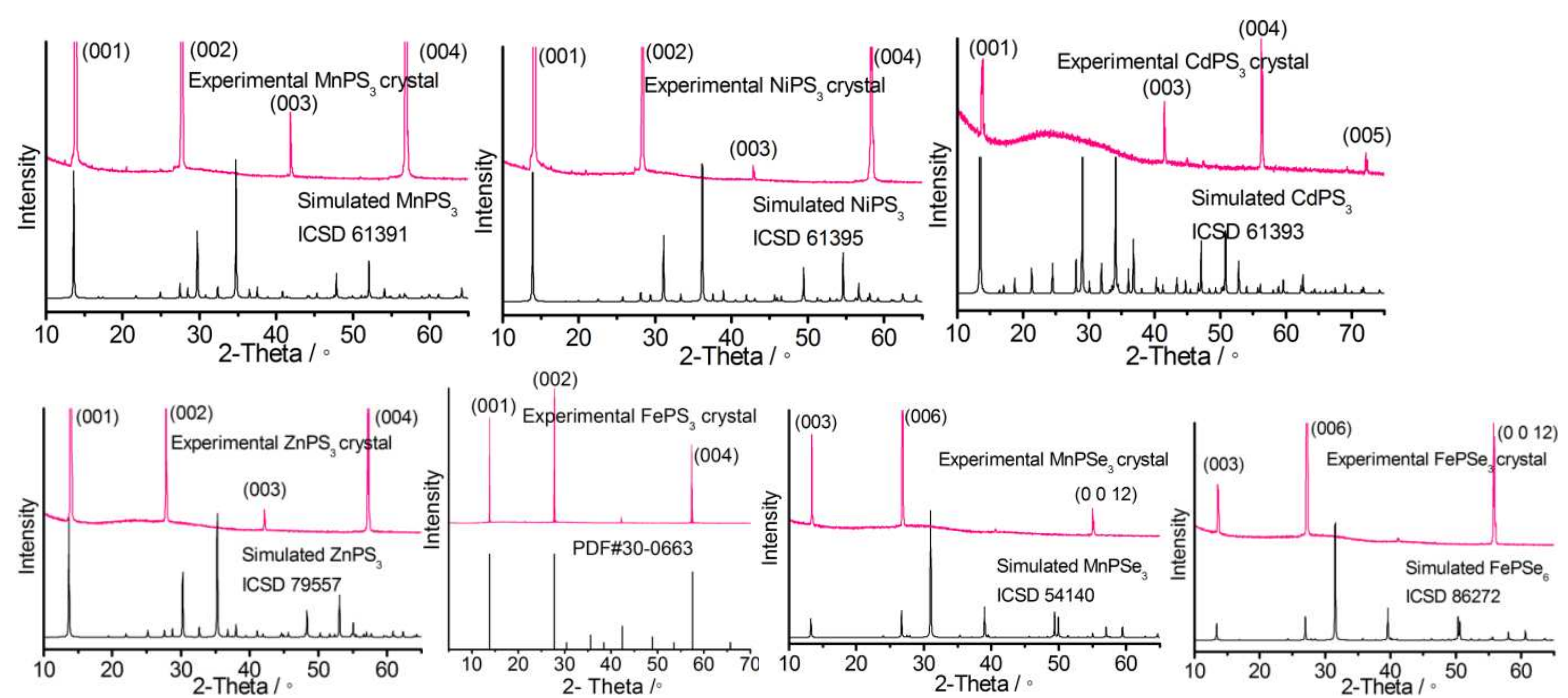

Fig. S1 The PXRD of the single bulk crystals. The simulated PXRD pattern was generated by Mercury 2.3 software from corresponding single crystal crystallography (cif).

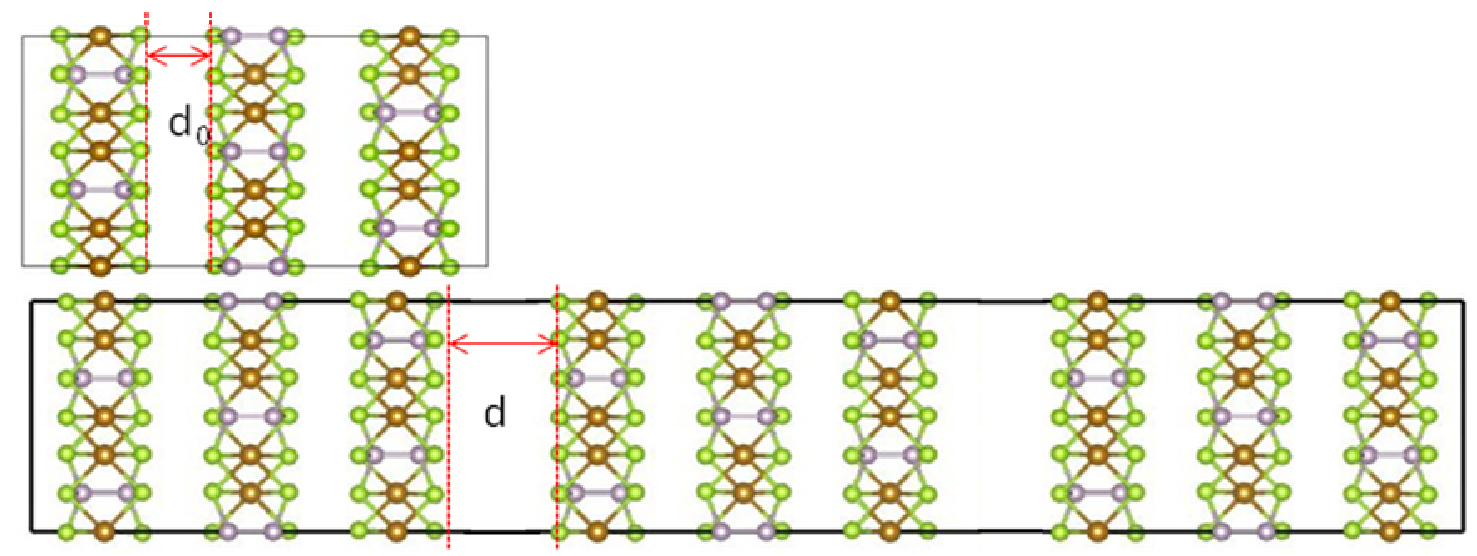

Fig. S2. The orginal interlayer distance $\left(d_{o}\right)$ and fractured $(d)$. The difference value is defined as separation.
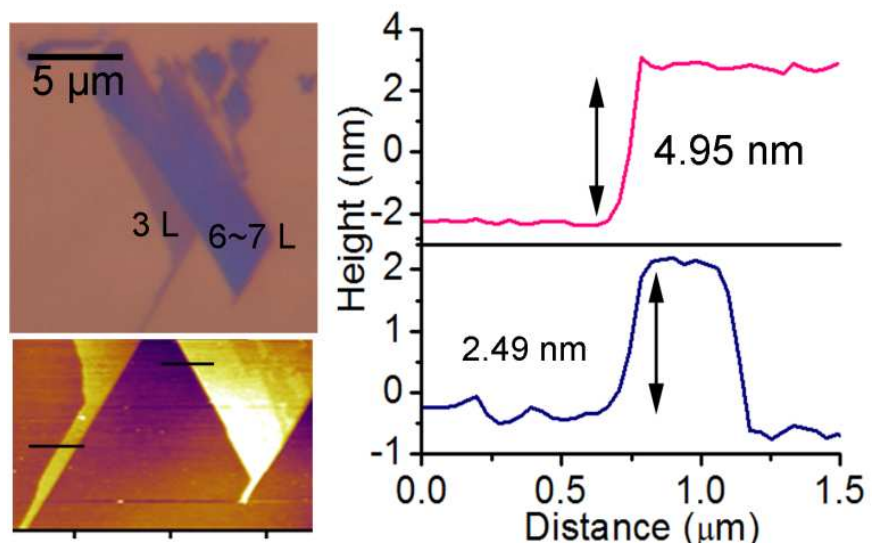

Fig. S3. The AFM of FePSe3 thin sample for Raman measurement.

Table S1. Some parametres and research of this series compounds. ${ }^{1-3}$

\begin{tabular}{|l|l|l|l|}
\hline Style & Compounds & Band gap reported & Remark \\
\hline
\end{tabular}




\begin{tabular}{|c|c|c|c|}
\hline \multirow[t]{5}{*}{$\mathrm{I}(C 2 / \mathrm{m})$} & $\begin{array}{l}\text { FePS3 } \\
\text { (ICSD 61392) }\end{array}$ & $1.5 \mathrm{eV}$ & $\begin{array}{l}\text { Intercalation, antiferromagnetism, } \\
\text { (2D Ising mode) }\end{array}$ \\
\hline & $\begin{array}{l}\mathrm{MnPS}_{3} \\
\text { (ICSD 61391) }\end{array}$ & $3.0 \mathrm{eV}$ & $\begin{array}{l}\text { Intercalation, antiferromagnetism, } \\
\text { (anisotropic Heisenberg model) }\end{array}$ \\
\hline & $\begin{array}{l}\mathrm{NiPS}_{3} \\
\text { (ICSD 61395) }\end{array}$ & $1.6 \mathrm{eV}$ & $\begin{array}{l}\text { Intercalation, antiferromagnetism, } \\
\text { (anisotropic Heisenberg model) }\end{array}$ \\
\hline & $\begin{array}{l}\text { CdPS3 } \\
\text { (ICSD 61393) }\end{array}$ & $3.5 \mathrm{eV}$ & $\begin{array}{l}\text { Intercalation } \\
\text { Structure transition }\end{array}$ \\
\hline & $\begin{array}{l}\mathrm{ZnPS}_{3} \\
\text { (ICSD 79557) }\end{array}$ & $3.4 \mathrm{eV}$ & Intercalation \\
\hline \multirow[t]{2}{*}{ II $(R \quad \overline{3})$} & $\begin{array}{l}\mathrm{FePSe}_{3} \\
\text { (ICSD 86272) }\end{array}$ & $1.3 \mathrm{eV}$ & $\begin{array}{l}\text { Intercalation } \\
\text { Antiferromagnetism }\end{array}$ \\
\hline & $\begin{array}{l}\text { MnPSe3 } \\
\text { (ICSD 54140) }\end{array}$ & $2.5 \mathrm{eV}$ & $\begin{array}{l}\text { Intercalation } \\
\text { Antiferromagnetism } \\
\text { Spinelectronic }\end{array}$ \\
\hline
\end{tabular}

1. Brec, R.; Schleich, D. M.; Ouvrard, G.; Louisy, A.; Rouxel, J., Physical-Properties of Lithium Intercalation Compounds of the Layered Transition Chalcogenophosphates. Inorg. Chem. 1979, 18, 1814-1818.

2. Balkanski, M., Spin-Dependent Light Scattering in Two-Dimensional Magnetically Ordered Systems. In New Horizons in Low-Dimensional Electron Systems, Aoki, H.; Tsukada, M.; Schlüter, M.; Lévy, F., Eds. Springer Netherlands: 1992; Vol. 13, pp 71-84.

3. Brec, R., Review on Structural and Chemical Properties of Transition Metal Phosphorus Trisulfides MPS3. In Intercalation in Layered Materials, Dresselhaus, M. S., Ed. Springer US: 1986; Vol. 148, pp 93-124. 